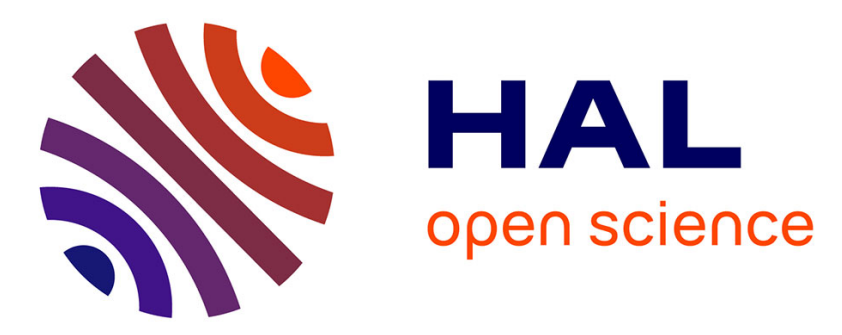

\title{
Que fait-on quand on nomme? Le handicap psychique face aux figures de la folie et de la maladie mentale
}

\author{
D. Moreau
}

\section{To cite this version:}

D. Moreau. Que fait-on quand on nomme? Le handicap psychique face aux figures de la folie et de la maladie mentale. Annales Médico-Psychologiques, Revue Psychiatrique, 2010, 168 (10), pp.770. 10.1016/j.amp.2010.09.012 . hal-00698864

\section{HAL Id: hal-00698864 https://hal.science/hal-00698864}

Submitted on 18 May 2012

HAL is a multi-disciplinary open access archive for the deposit and dissemination of scientific research documents, whether they are published or not. The documents may come from teaching and research institutions in France or abroad, or from public or private research centers.
L'archive ouverte pluridisciplinaire HAL, est destinée au dépôt et à la diffusion de documents scientifiques de niveau recherche, publiés ou non, émanant des établissements d'enseignement et de recherche français ou étrangers, des laboratoires publics ou privés. 


\section{Accepted Manuscript}

Title: Que fait-on quand on nomme? Le handicap psychique face aux figures de la folie et de la maladie mentale

Author: D. Moreau

PII: $\quad$ S0003-4487(10)00324-0

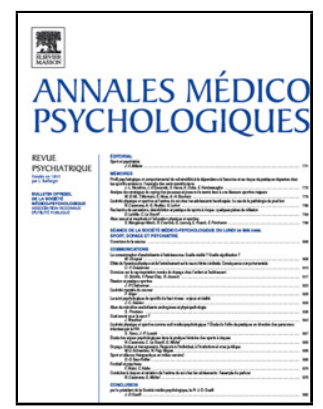

DOI: $\quad$ doi:10.1016/j.amp.2010.09.012

Reference: $\quad$ AMEPSY 1254

To appear in: $\quad$ Annales Médico-Psychologiques

Please cite this article as: Moreau D, Que fait-on quand on nomme? Le handicap psychique face aux figures de la folie et de la maladie mentale, Annales mediopsychologiques (2010), doi:10.1016/j.amp.2010.09.012

This is a PDF file of an unedited manuscript that has been accepted for publication. As a service to our customers we are providing this early version of the manuscript. The manuscript will undergo copyediting, typesetting, and review of the resulting proof before it is published in its final form. Please note that during the production process errors may be discovered which could affect the content, and all legal disclaimers that apply to the journal pertain. 
Communication

Que fait-on quand on nomme?

\title{
Le handicap psychique face aux figures de la folie et de la maladie mentale \\ Madness, mental illness, "psychic handicap":
}

The issues of the categories used to designate the mental troubles

\section{Moreau}

Delphine Moreau, GSPM-EHESS Groupe de Sociologie Politique et Morale, Ecole des Hautes Etudes en Sciences Sociales, 10 rue Monsieur-le-Prince, 75006 Paris, France 34 bd de Sébastopol 75004 Paris, France

0689207787

Adresse email : delphmoreau@free.fr

\section{Résumé}

La diffusion récente de la notion de handicap psychique ajoute une figure à la désignation des troubles mentaux. Nous esquissons ici quelques-uns des enjeux de cette nouvelle catégorisation. Après la notion de maladie, celle de « handicap psychique » apparaît comme une manière de désigner les troubles mentaux d'une manière moins inquiétante que la figure de la folie. Elle permet de plus de rendre visibles et prendre en compte leurs conséquences sociales spécifiques. Pour autant, le handicap reste porteur de son stigmate propre.

Mots clés : Folie ; Handicap mental ; Handicap psychique ; Maladie mentale ; Stigmate

\begin{abstract}
The concept of "psychic handicap" has appeared recently in the French context. This article outlines some of the challenges this new categorization. The concept of "psychic handicap" is analysed as a way less pejorative than "madness" and "illness” to designate the mental troubles. It opens to a better consideration of the social needs of people with mental illness. However, the concept of disability bears his own stigma.
\end{abstract}

Keywords: Madness; Mental handicap; Mental illness; Psychic handicap; Stigma 
La loi du 11 février $2005^{1}$ a été décrite, avec un effet performatif, comme la reconnaissance officielle de la notion de handicap psychique ${ }^{2}$. Promue notamment par des associations de familles et de patients, celle-ci a cependant mis un certain temps à s'imposer et continue de rencontrer résistances et critiques. Sans décrire ici les ramifications fines des qualifications possibles des troubles psychiques ${ }^{3}$, nous nous attacherons à dégager certains enjeux de cette nouvelle catégorisation en la confrontant à ces autres figures génériques que sont la folie et la maladie, dans ses deux versants psychodynamique et biologique, et, au sein du handicap, à la notion de handicap mental.

\section{Catégoriser : définir une situation pour agir ou enfermer dans un mot?}

La qualification des troubles engage la « place sociale » qui leur est donnée. Différents auteurs ont souligné que la psychiatrie moderne s'était constituée par le fait de traiter la folie de manière distincte d'autres types d'écarts à la norme (oisiveté, vagabondage, criminalité...), et, d'autre part, de la saisir comme maladie, en réduisant toute interprétation mysticoreligieuse. Foucault a pu lire, derrière la légende dorée du «déchaînement des fous de Bicêtre », certes une sortie du "grand renfermement », mais via une nouvelle exclusion par leur relégation dans l'asile et leur réduction au silence sous le rationalisme médical [5]. Swain et Gauchet y ont vu, en revanche, la reconnaissance démocratique du semblable dans le fou, le traitement moral promu par Pinel puis son élève Esquirol reposant sur le postulat de la part de raison préservée chez leurs patients [8]. Donner aux personnes souffrant de troubles psychiques une place spécifique signifie-t-il leur donner une place adaptée, qui répond à leur mal propre, ou ne risque-t-il pas de conduire à une forme d'exclusion ou d'étiquetage ?

Le risque d'enfermement dans une catégorie est l'une des raisons avancées aujourd'hui par les psychiatres contre la notion de handicap psychique. Il est également mobilisé pour refuser de donner un diagnostic (par-delà la difficulté parfois de poser celui-ci), craignant en nommant de provoquer une mise à l'écart sociale de la personne et de sceller son destin, quand la présence de troubles psychiques graves, même lorsqu'elle est clairement identifiée (ce qui n’est pas toujours le cas), ne préjuge pas du devenir de la personne, de

\footnotetext{
${ }^{1}$ Loi $n^{\circ}$ 2005-102 du 11 février 2005 pour l'égalité des droits et des chances, la participation et la citoyenneté des personnes handicapées.

${ }^{2}$ De fait, sans mentionner l'expression de handicap psychique, le texte cite l'altération d'une ou plusieurs fonctions psychiques comme « facteur » de handicap [2].

${ }^{3}$ Sur l'articulation entre qualification des troubles et intervention pratique [6], ainsi que les travaux d'A. Béliard et J.-S. Eideliman [1].
} 
l'évolution de sa pathologie comme de son avenir social. De plus, donner ce diagnostic de façon intempestive risquerait de brutaliser le patient et pourrait renforcer son déni.

Cependant, ne pas nommer peut être source d'angoisse ou de difficultés pour ceux qui sont confrontés aux troubles. «Poser un mot », c’est aussi identifier ce qui se passe et le registre de réponse pratique exigée. Le fait de ne pas livrer un diagnostic ne signifie pas que les psychiatres n'en posent pas, au moins à titre d'hypothèse de travail, ou qu'ils ne se réfèrent pas à de grandes catégories pathologiques, au moins pour établir un traitement et la conduite à tenir. De la même façon, les proches ont besoin de situer ce qui se passe, à plusieurs niveaux : pour comprendre une conduite qui leur échappe, pour rendre compte de l'inquiétude qui les saisit, pour voir reconnu le niveau de gravité de la situation tel qu'ils le perçoivent, éventuellement justifier leur intervention d'aide ou de contrainte pour amener au soins, et les aider à définir leurs actions futures. En deçà de la détermination d'un diagnostic précis est en jeu la confirmation qu'il s'agit d'un trouble d'ordre psychique. Nombre d'interprétations peuvent se succéder en amont, de la crise d'adolescence à l'usage de drogue, des «mauvaises fréquentations » à un caractère paresseux ou colérique, ce qui est source de conflit entre les proches sur l'attitude à avoir entre blâme moral et inquiétude pour la santé. L'identification d'un problème psychiatrique est souvent tardif, lorsque les autres interprétations échouent à rendre compte de ce qui se passe et que la gravité des faits exige une intervention [6].

\section{Quand le spectre de la « folie » rôde...}

L’interprétation en termes psychiatriques est d'autant plus repoussée que la « folie » continue d’être la figure effrayante qui y est associée, et que les personnes concernées comme leurs proches cherchent à conjurer lorsque les troubles apparaissent, et ce malgré le développement du discours biomédical et la diffusion des références au « psy » dans le grand public $[3,4]$.

Le modèle de la maladie offre une première manière de conjurer ce spectre, notamment dans sa version biologique: il représente en effet un modèle descriptif et explicatif unifiant, face à une série de conduites et d'événements troublants ; il ouvre l'espoir, en partie fantasmatique, de guérir les troubles, ou, tout au moins, de les réguler. Des proches rencontrés font ainsi un parallèle avec le diabète, comme modèle «non stigmatisant » d'une maladie chronique pour laquelle il faut prendre un traitement à vie. 
De plus, par opposition aux interprétations psychodynamiques, le modèle biologique suspend l'idée d'une «culpabilité » des parents dans la genèse des troubles, en postulant des relations de cause à effet biochimiques. Certains parents préfèreront cependant conserver une hypothèse psychodynamique qui donne un «sens » aux troubles et les inscrit dans une histoire.

Le modèle de la maladie a aussi pour intérêt de suspendre la responsabilité de la personne elle-même: ses conduites ne sont pas de simples conduites déviantes, ou l'expression d'un manque de volonté dont elle pourrait être tenue pour responsable.

Certains psychiatres n’hésitent cependant pas à employer le mot de «folie », la psychiatrie devant selon eux «accueillir la folie », y compris dans sa part terrifiante, mais aussi dans son caractère pleinement humain. La notion de handicap psychique peut apparaître dans ce cadre comme une tentative d'euphémisation des troubles, ainsi qu'une manière de réduire les potentialités des patients.

\section{Handicap « psychique " versus « mental "}

Aux yeux de certains proches, le «handicap psychique » a précisément pour mérite d'être «moins stigmatisant» et moins effrayant que la «folie » ou certains termes de diagnostic. En outre, la promotion d’une notion spécifique de « handicap psychique » permet d'écarter la « dangereuse » proximité avec la déficience mentale. La réaction consistant à se distinguer d'un autre stigmate proche se rencontre fréquemment dans un groupe stigmatisé : à la fois pour ne pas subir les effets indus du voisinage et risquer d'être confondu avec lui, mais aussi afin de mettre en évidence ce qu’on a d' «intact », de préservé, de «normal », en reprojetant l' « infériorité » ou l' « anormalité » sur la catégorie dont on s’écarte.

De plus, l'un des enjeux centraux de la reconnaissance du « handicap psychique » est de caractériser les troubles d'une manière qui légitime la prise en charge " collective » des difficultés qu'ils entraînent et qui l'inscrit dans l'architecture de la solidarité organisée par l'État ${ }^{4}$. Certes, les personnes dont le trouble avait de forts retentissements dans leur vie sociale pouvaient déjà obtenir la reconnaissance d'un handicap ouvrant droit à un travail en milieu protégé, à un hébergement dans une structure spécifique et/ou à une allocation. Mais tous ceux qui sont confrontés de près à ces troubles dénoncent le manque criant de structures adaptées. Les conséquences des troubles mentaux étant mal connues, elles sont souvent

\footnotetext{
${ }^{4}$ F. Chapireau montre le fort cloisonnement des filières sociales, médico-sociales et sanitaires [2]
} 
inégalement reconnues. L’Unafam (Union Nationale des Amis et Familles de Malades psychiques) affirmait ainsi explicitement que cette notion avait pour but de « faire exister une population », c’est-à-dire de la rendre visible, et de la rendre visible comme devant être aidée.

\section{Conclusion}

Il existe ainsi des hiérarchisations différenciées de ces catégories : folie, maladie, handicap, et qui peuvent évoluer. Dans le processus qui conduit à adopter une définition de la situation, puis éventuellement à en changer, interagissent l'acceptabilité de cette définition et sa mise à l'épreuve par l'évolution de la situation elle-même (par exemple, la réitération d'épisodes conduisant à des hospitalisations, l'échec à « tenir » un emploi, etc.). Il faut noter que le repérage de ces figures n’implique pas le présupposé qu’elles seraient homogènes. Il existe toute une multiplicité d’interprétations, d'appropriations et de découpages de ces figures. De plus, les différentes catégories ne recouvrent pas les mêmes enjeux selon la position de chaque acteur: la personne elle-même, les soignants, les proches, ou des personnes n'ayant pas d'expérience directe des troubles psychiques. ${ }^{5}$

La catégorie de handicap éloigne la figure du «fou dangereux imprévisible à enfermer »; elle souligne et objective les difficultés sociales vécues et appelle aide et compensation; elle ouvre le registre de la sollicitude. Moins stigmatisante, elle reste cependant mal acceptée, notamment en France, où elle continue d'être associée à une déficience définitivement attachée à la personne. Ce qui est reproché au handicap, c’est son caractère figeant, malgré les évolutions conceptuelles dont témoigne la loi de 2005 [2]. La maladie offre une perspective plus évolutive et ouvre sur la possibilité de soins. Le moment de reconnaissance du handicap peut ainsi être vécu comme l'entrée dans une catégorie dégradée, notamment du point de vue des personnes elles-mêmes.

\section{Conflit d'intérêt : à compléter par l'auteur}

\section{Références}

[1] Béliard A, Eideliman JS. Aux frontières du handicap psychique : genèse et usages des catégories médico-administratives. Revue Française des Affaires sociales 2009:99-117.

\footnotetext{
${ }^{5}$ Ce qu'on ne peut réduire à l'opposition entre profanes et professionnels : la catégorie de profane ne permet pas de faire droit à l'expérience directe de la maladie de ceux qui la vivent à la première personne ou à travers une relation proche et prolongée; et ce serait postuler chez les professionnels des représentations pures de leur propre expérience sociale [7]
} 
[2] Chapireau F. Handicap psychique : confirmation ou mutation du handicap à la française. Psychiatrie. Encyclopédie Médico-Chirurgicale 2006. 37-910-A-10.

[3] Dodier N, Rabeharisoa V. Les transformations croisées du monde «psy» et des discours du social. Politix 2006:9-22.

[4] Ehrenberg A, Lovell A. La maladie mentale en mutation. Psychiatrie et société. Paris: Odile Jacob; 2001.

[5] Foucault M. Histoire de la folie à l'âge classique ( $1^{\mathrm{e}}$ éd. Plon, 1961). Paris: Gallimard; 1976.

[6] Moreau D. Faire interner un proche ? Le travail sur l'autonomie en contexte de troubles psychiques. CNAF Dossiers d'Études 2007;9:1-65.

[7] Sinding C. Catégories des médecins, catégories de malades (Commentaire). Sciences Sociales et Santé 1999;17:23-9.

[8] Swain G, Gauchet M. La pratique de l'esprit humain : l'institution asilaire et la révolution démocratique. Paris: Gallimard; 1980.

\section{Discussion}

Dr R. Lécuyer - Merci de ce remarquable essai de clarification. Pour compliquer un peu les choses, je signale que l'Association «Nous aussi », regroupant les personnes handicapées mentales elles-mêmes, souhaiterait qu'on les qualifie de «Personnes en situation de handicap intellectuel ».

Dr C. Houssou - Comment différenciez-vous en pratique le trouble mental du trouble psychique?

Réponse du Rapporteur - Au Dr R. Lécuyer - Votre première remarque illustre le fait que le choix d’une qualification pour désigner un problème ne se réfère pas à une hiérarchie absolue entre les catégorisations ni entre les phénomènes stigmatisés, mais à la manière dont chacun se les approprie et les inscrit dans un système d'associations et d'oppositions qui prend aussi sens à partir du point de vue où l'on se place. Est-ce que cette notion de « handicap intellectuel » vise de manière réciproque à écarter tout risque de confusion avec les troubles mentaux? Ces volontés symétriques de personnes souffrant de troubles psychiques et d'un handicap intellectuel de ne pas être confondues les unes avec les autres 
manifesteraient alors on ne peut plus clairement le stigmate dont chacune est porteuse et le phénomène de distinction d'un stigmate proche pour mettre en avant ce que l'on considère comme « préservé ».

Au Dr C. Houssou - « Psychique » est aujourd’hui le terme qui tend à être préféré à «mental », notamment en association avec la notion de handicap psychique. Dans les expressions «trouble mental» et «trouble psychique», il me semble que ces adjectifs désignent les mêmes phénomènes. Le sociologue ne cherchera d’ailleurs pas à distinguer luimême les troubles mais s'attachera plutôt à observer les opérations de qualifications opérées par les acteurs dans des situations données et à en dégager les enjeux. 